\title{
Properties of Images in LSB Plane
}

\author{
Kaustubh Choudhary \\ Scientist, Defence Research and Development Organisation (DRDO), Naval College of Engineering, Indian \\ Naval Ship Shivaji, Lonavla, Maharashtra, India
}

\begin{abstract}
Each pixel of an Image (assuming 24 bit BMP) is stored in the three 8 bits corresponding to three colors. Bit Plane Slicing is the technique of breaking image into 8 planes corresponding to 8 different bit positions. The bit plane image corresponding to the plane of the most significant bit (MSB) has the maximum contribution to the total image and forms the majority of the visually significant image data and planes corresponding to other lower bit positions contribute only the subtle details of the image. And the bit-plane image corresponding to the LSB of the RGB value has the minimum contribution of only 1 out of total of 255 Intensity level to the total image. In spite of this the LSB Plane of the image is not insignificant. In fact it is used in Image Steganography for hiding secret information in the images. Image based Steganography is a potent tool used by Terrorists and Criminal organizations for securely broadcasting, dead-dropping and communicating malicious secret messages over the internet by hiding them in the images. Our cyber space is full of such mala-fide images containing hidden secret information. The most difficult aspect of tracking such malicious images is the lack of efficient and fast algorithms for identifying and isolating them from the bulk of innocent images. Analysis of the pixels in the LSB Plane of the image is very effective technique of identification of Distributing Spatial Domain Steganographic Algorithms. In this paper the properties of Information Pixels pixel present in the LSB Plane of any stego image generated by Distributing Algorithms is analyzed in detail. These properties will be very useful in identification of malafide stego images lurking in our cyber space. All the images and tables used in this paper are generated using MATLAB OImage Processing Tool Box.
\end{abstract}

Key Words: Bit Plane Slicing, Cyber Crime, Distributing Steganographic Algorithms. Global Terrorism, Image Steganalysis, Multicolor LSB Transform, SDT based Image Steganography.

\section{INTRODUCTION}

Digital image is made up of discrete picture elements known as pixels and the pixel information (Red, Green and Blue intensity levels) of every pixel is stored in fixed sized memory space corresponding to each pixel. The size of memory and the way the pixel information is stored depends upon the format of the image. For example in a 24 bit BMP image each pixel is stored as three 8 bits corresponding to the intensity levels of the three primary colors i.e. RGB values while other formats may use a entirely different model for storing the pixel information but all of them store the pixel information in the memory corresponding to each pixel. So for the sake of simplicity a 24 bit BMP Image is assumed for explaining Bit Plane Slicing. The digital image is explained elaborately in elaborately explained in section 2 of [1].

Each pixel of a 24 bit BMP Image is stored in the three 8 bits corresponding to three colors. In other words pixel information consists of 8 bit RGB values corresponding to each and every pixel. So the entire image can be thought to be consisting of eight different images or planes corresponding to 8 different bit positions in the RGB value. Thus these 8 different bit-planes highlight the contribution of each of the eight different bit position of the RGB Value to the total image. In other words the entire single image can be sliced in to 8 images-planes corresponding to each bit of the RGB value and this process is called as Bit Plane Slicing. The bit plane image corresponding to the plane of the most significant bit (MSB) has the maximum contribution to the total image and forms the majority of the visually significant image data and therefore almost represents the total image. The bit-plane images corresponding to other lower bit positions contribute only the subtle details of the image. Whereas the bit-plane image corresponding to the LSB of the RGB value has the minimum contribution of only 1 out of total of 255 Intensity level to the total image. Thus LSB plane of the Image appears black (some variation in black shade can be seen if the image is highly enlarged) due to negligible contribution (of only 1 out of 255 intensity levels) to the total image and is insignificant from the image processing point of view. But this insignificant appearing LSB plane of the Image is highly useful in Steganographic techniques for hidding the secret information in the images. In fact the LSB Plane of the image has maximum concentration of the secret information in the image.

\subsection{Steganography}

Image based steganography is a dangerous technique of hiding secret messages in the image in such a way that no one apart from the sender and intended recipient suspects the existence of the message. It is based on invisible communication and this technique strives to hide the very presence of the message itself from the 
observer. As a result it has been used more frequently by various criminal and terrorist organizations than anybody else.[2][3][4] Various agencies even claim that 9/11 attacks have been masterminded and planned using image based steganography [5]. Image Steganography offers numerous advantages to the terrorists like Anonymity, Electronic Dead Dropping, Secure Broadcasting and above all very high Secrecy and Security (explained in detail in Section 5.2.1 of [1]). Thus an innocent looking digital image on any Web Portal, Online Auction Site or even a Social Networking Site may be probably hiding a malicious and deadly terrorist plan or any other significant criminal Information. The Steganalysis is the technique of identifying such malicious Stego-images (original image which is used for hiding data is called the Cover-Image whereas the image obtained after inserting the Secret Information in it is called Stego Image) from the bulk of innocent images. Considering the voluminous bulk of images flowing every day through the Internet and amount of time and Computation Cost required for analyzing the Image the very first step of identifying an innocent looking Image as a Stego Image becomes the most challenging part of any Steganalysis procedure. It is because we do not have any foolproof method for crisply identifying a steganographic signature in the innocent looking stego-image. The basic of image based steganography is explained in detail in [1].

Properties of stego-images (images containing information) depends upon the properties of those pixels in the stego-image which are storing the information- referred as Information Pixels. In other words the properties of stego-images become different from the innocent image (image without information) due to deformation produced in certain pixels of stego-image due to embedding of information in those pixels.

As a result every steganographic algorithms has a unique signature. As mentioned in [6] the most spatial domain steganographic algorithms can be broadly classified in to two types- Distributing Steganographic Algorithms and Concentrating Steganographic Algorithms. In other words all spatial domain steganographic algorithms either embeds the information in the Least Significant Bits of the pixel or changes the entire color code of the pixel by inserting information in more than 2 bits of the pixel. In former algorithms large number of pixels are required for inserting information because only one or two LSB is available from every pixel and hence known as Distributing Type while in the latter algorithm the entire information can be stored in very few pixels because large numbers of bits are available from every pixel for storing information and hence called as Concentrating Type.

Since the amount of change produced in the pixels by Concentrating Algorithms is very large so identification of stego images generated by Concentrating stego algorithms is relatively easier and occasionally can be even identified by the visual clues only. This can be clearly seen from the Fig 1 of [6]. At the same time the pixel aberration based analysis (explained in detail in [6] [7] [8] is easily capable of identifying stego images generated by Concentrating Stego Algorithms. But in case of Distributing Steganographic Algorithms the change brought in every pixel is very low and as a result their identification becomes a very challenging task. As a remedy a technique based on Multicolor LSB Transform was proposed in Section 2.2 of [6] for the identification of Distributing Stego Algorithms. In this paper the properties of these information pixels (present in Multicolor LSB Transform) are analyzed in detail. These properties can be very useful in determining suspicion value (a number associated with the image which is bigger for the images containing information and smaller for innocent images.) of any given image and were partially used in [9] to generate Distributing Suspicion Value $\Lambda(\mathrm{M})$ for any Image $\mathrm{M}$.

\subsection{LSB Plane has Information}

Since the LSB of the RGB values of the image hides the secret information so the pixels forming the LSB Plane of the Image either represent the hidden information or the LSB Component of the total Image. [1][6][9] In other words the image corresponding to the contribution of the LSB to the stego-image also contains the secret information. But the intensity levels for any of the R, G or B value of any image in LSB plane is at $\max 1$ which is negligibly small when compared with total of 255 intensity levels. As a result the image in its LSB Plane appears black. So although the LSB Plane of the Stego-Image has maximum concentration of secret information but due to its dark black color we are unable to see the information in the image. As a remedy if the contrast of the LSB Plane of the image can be increased, then we can easily see the information stored in the image. A technique used here is that if any of the R, G or B value of the LSB Plane of the image is 1 then it is replaced by the intensity level of 255 whereas the intensity level of 0 is left unchanged. So the new image obtained has R, G or B value of every pixel as either 255 (if the corresponding true LSB value was 1) or else it remains 0 . Therefore the modified LSB Plane of the image will appear multicolored and each pixel of this image will have one of the seven possible colors ranging from three primary colors (Red, Green and Blue), four other colors obtained by their combination i.e. Yellow $(R+G)$, Magenta $(R+B)$ and Cyan $(B+G)$, White $(R+G+B)$ and Black indicating absence of any of the three primary color components. In Figure 1 all these 6 colors (Excluding Black) are shown as a combination of 3 primary colors and also a section of Multicolored LSB Plane of some Image is shown. It can be clearly seen that any pixel of the Multi-Colored LSB plane has only one of those six colors or else it is black in color. Hence hereafter in this 


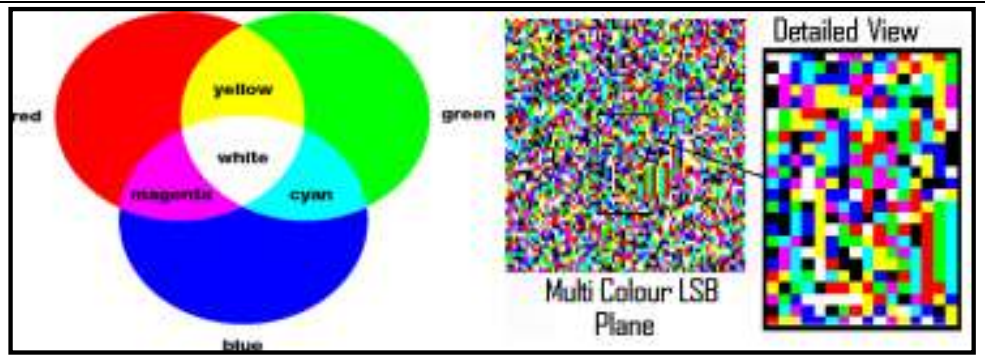

Fig 1 Three Primary Colors and Their Combinations and Multicolored LSB Plane of some Image

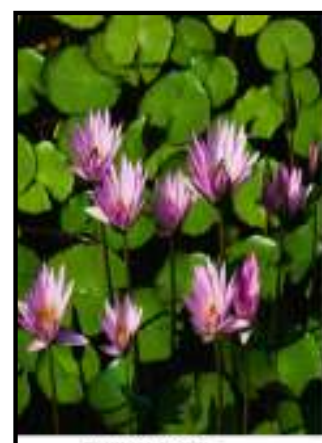

ORIOMLL BMCE

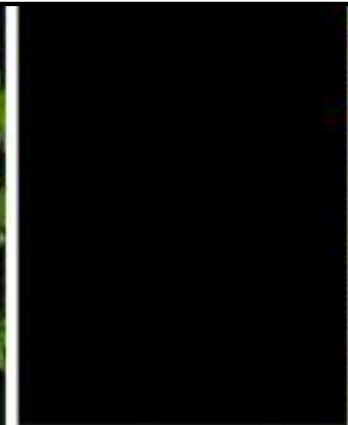

TRUE LSB PLANF UF THE BWEE

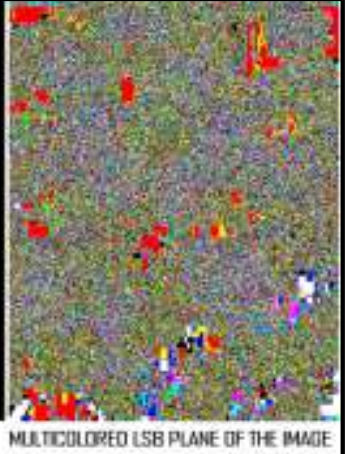

MULCOLTERO LSB PLANE OF THE MANE

Fig 2 Original Image and Corresponding True LSB and Multicolored LSB Plane (width reduced by 60\%)

paper this modified LSB Plane of the image will be referred as Multicolored LSB Plane of the Image. Due to the presence of 7 colors corresponding to each of the LSB value of the image the presence of information in the LSB of the Image can be easily traced even by the visual clues only. In Figure 2 the LSB Plane and Multicolored LSB Plane is show for the same Image.

\section{PRELIMINARIES AND DEFINITIONS}

In order to analyze the properties of the information pixels we need to crisply and formally define certain concepts to be used in this analysis. These concepts are briefly summarized as definitions in this section. These preliminary concepts are derived from the concepts mentioned in [6], [7], [8] and [9].

\section{Definition 1 (Image)}

Every digital image is collection of discrete picture elements or pixels. Let $M$ be any digital image with $\mathrm{N}$ pixels. So any particular pixel of image $\mathrm{M}$ is represented as $\mathrm{M}(\mathrm{z})$ and $\mathrm{z}$ can be any value from 1 to $\mathrm{N}$. This $\mathrm{M}(\mathrm{z})$ can be a gray level intensity of the pixel in gray scale image or RGB or YCbCr value of the pixel in a color Image. The the individual RGB components of the pixel $M(z)$ in image $M$ is represented as $M^{R}(z), M^{G}(z)$ and $\mathrm{M}^{\mathrm{B}}(\mathrm{z})$ respectively. Thus $\mathrm{M}(\mathrm{z})$ can be a set $\left\{\mathrm{M}^{\mathrm{R}}(\mathrm{z}), \mathrm{M}^{\mathrm{G}}(\mathrm{z}), \mathrm{M}^{\mathrm{B}}(\mathrm{z})\right\}$ or equivalent gray scale representation or $\left(\mathrm{M}^{\mathrm{R}}(\mathrm{z})+\mathrm{M}^{\mathrm{G}}(\mathrm{z})+\mathrm{M}^{\mathrm{B}}(\mathrm{z})\right) / 3$. But it is always better to consider each $\mathrm{R}, \mathrm{G}$ and $\mathrm{B}$ components individually because the averaging effect cause loss of vital steganographic information. Further $\langle\{\mathrm{M}\}, \mathrm{m}>$ is multiset of

Image $\mathrm{M}$ such that $\mathrm{M}(\mathrm{z}) \in\{\mathrm{M}\}$ for every $\mathrm{z}=1$ to $\mathrm{N}$ and $\mathrm{m}$ is a vector corresponding to the occurrence or count of every element $M(z)$ in $\{M\}$. Mathematically an image $M$ with $N$ pixels is:

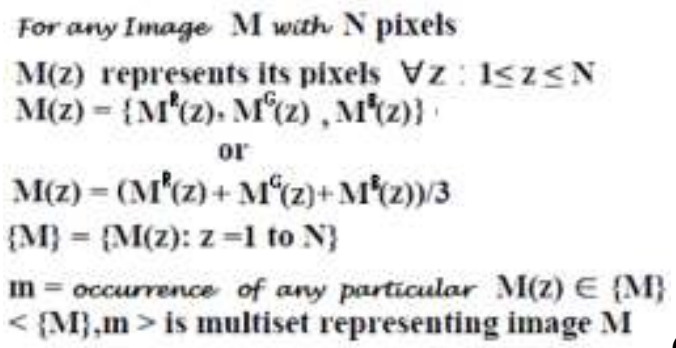

\section{Definition 2 (Cardinality or Size of Image)}

Any Image $M$ consists of certain number of pixels. So any particular pixel of image $M$ is represented as $\mathrm{M}(\mathrm{z})$ and $\mathrm{z}$ can be any value from 1 to total number of pixels in the image. The cardinality or the size of the image $M$ is the total number of pixels present in the image and represented as $n(M)$. So any Image $M$ has $n(M)$ pixels.

Thus 


$$
\mathbf{M}=\bigcup_{\mathrm{z}=1}^{\mathbf{n}(\mathbf{M})} \mathbf{M}(\mathbf{z})
$$

\section{Definition 3 (Component of an Image)}

Any sub part of an Image is a component of the image. In other words any Image $\mathrm{M}$ can be broken down into pixel groups (or clusters) and each such cluster forms a component of the image and is identified by its unique set of pixels. Thus in a Image M the Pixels $M(z)$ from $z=1$ to $n(M)$ are the elements of the image $M$ and the subsets of the image $M$ are composed of some of those pixels ( $M(z)$ from $z=1$ to $n(M)$ ) and thus forms the components of the image. Thus if the image $\mathrm{M}$ is broken in to $\mathrm{K}$ components then any component $\mathrm{M}^{\mathrm{i}}$ of the image $\mathrm{M}$ is mathematically explained as:

$$
\begin{gathered}
\mathbf{M}^{\mathrm{i}} \subset \mathbf{M} \text { and } \forall \mathbf{i}: \mathbf{1} \leq \mathbf{i} \leq \mathbf{K} \\
\mathbf{M}=\bigcup_{\substack{\mathbf{M}^{\mathbf{i}} \subset \mathbf{M} \\
\forall \mathbf{i} \mathbf{1} \leq \mathbf{i} \leq \mathbf{K}}} \mathbf{M}^{\mathbf{i}}
\end{gathered}
$$

Also for every component $M^{i}$ of the image $M$ the $M^{i}(z)$ represents the pixels of the component $M^{i}$ and $n\left(M^{i}\right)$ represents the number of pixels in $\mathrm{M}^{\mathrm{i}}$.

\section{Definition 4 (Neighborhood or Locality of Pixel)}

If $\ell(M(z))$ is said to be set of neighboring pixels of any pixel $M(z)$ in image $M$. Then any $n_{i} \in \ell(M(z))$ will be such that $d\left(\mathrm{n}_{\mathrm{i}}, \mathrm{M}(\mathrm{z})\right) \leq \lambda$ where $d$ is a function which calculates distance (can be Euclidean, City-Block, Chess Board or any other type depending upon the steganographic algorithm) between its inputs (ie $n_{i}$ and $\mathrm{M}(\mathrm{z})$ ) and $\lambda$ is measurement of degree of neighbourhood and should be minimum (Generally equal to 1 pixel) but also depends upon the steganographic algorithm used. Mathematically this can be represented as:

$$
\left.I(\mathrm{M}(\mathrm{z}))=\{\mathrm{M}(\mathrm{x}): \mathrm{M}(\mathrm{x}) \in \mathrm{M} \text { and } d(\mathrm{M}(\mathrm{x}), \mathrm{M}(\mathrm{z})) \leq \lambda\}_{4}\right)
$$

An arbitrary pixel $\mathrm{Y}$ is shown with its neighbors $\mathrm{P}, \mathrm{Q}, \mathrm{R}, \mathrm{S}, \mathrm{T}, \mathrm{U}, \mathrm{V}$ and $\mathrm{W}$. We represent this pixel $\mathrm{Y}$ as $\dot{Y}$ in mathematical notation. Thus $\ell(\dot{Y})=\{\mathrm{P}, \mathrm{Q}, \mathrm{R}, \mathrm{S}, \mathrm{T}, \mathrm{U}, \mathrm{V}, \mathrm{W}\}$ is set of neighboring pixels of pixel $\mathrm{Y}$. Here $\lambda=1$ and distance function $d$ calculates Chess Board Distance.

\begin{tabular}{|l|l|l|}
\hline $\mathrm{P}$ & $\mathrm{Q}$ & $\mathrm{R}$ \\
\hline $\mathrm{S}$ & $\mathrm{Y}$ & $\mathrm{T}$ \\
\hline $\mathrm{U}$ & $\mathrm{V}$ & $\mathrm{W}$ \\
\hline
\end{tabular}

Pixel Y

Definition 5 Multicolored LSB Transform

Multicolor LSB Transform of any Image M is represented as $\mathrm{M}_{\mathbf{L S B}}$ and Corresponds to the Multicolor LSB Plane of the image M.Thus corresponding to the pixel M(z) of the image $M$ the pixel in $M_{L S B}$ is represented as $\mathrm{M}_{\mathbf{L S B}}(\mathrm{z})$. The relation between $\mathrm{M}(\mathrm{z})$ and $\mathrm{M}_{\mathbf{L S B}}(\mathrm{z})$ is explained mathematically in (5).

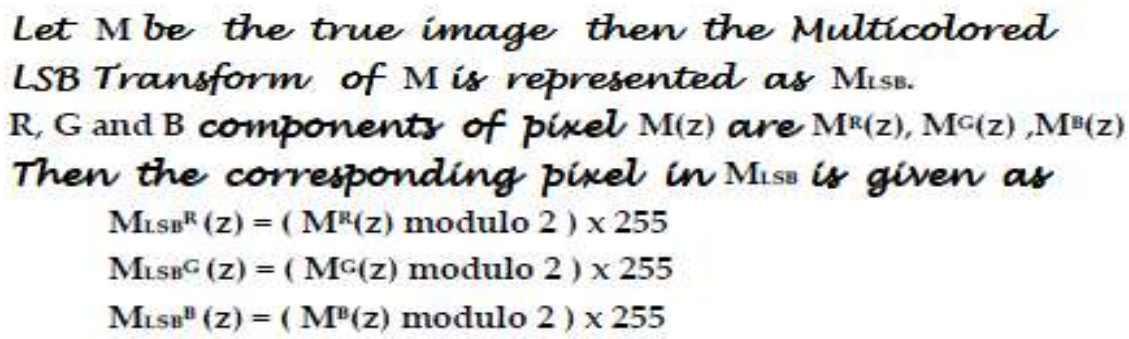

\section{Definition 6 (Deviation in the Multicolored LSB Transform)}

In [6] and [9] it was concluded that any statistically significant Component (at least more than $50 \times 50$ pixels) of Multicolored LSB Transform of an innocent image has nearly equal distribution of Red, Green and Blue Components. The Degree of Deviation in Multicolored LSB Transform of the image is a quantifier that measures the amount of deviation from equal distributions of the red, green and blue components in the image. This degree of deviation in Multicolored LSB Transform of the image $\mathbf{M}_{\mathbf{L S B}}$ is represented as $\mathfrak{D}\left(\mathbf{M}_{\mathbf{L S B}}\right)$. Algorithm for determining $\mathfrak{D}\left(\mathbf{M}_{\mathbf{L S B}}\right)$ is given in (6). 


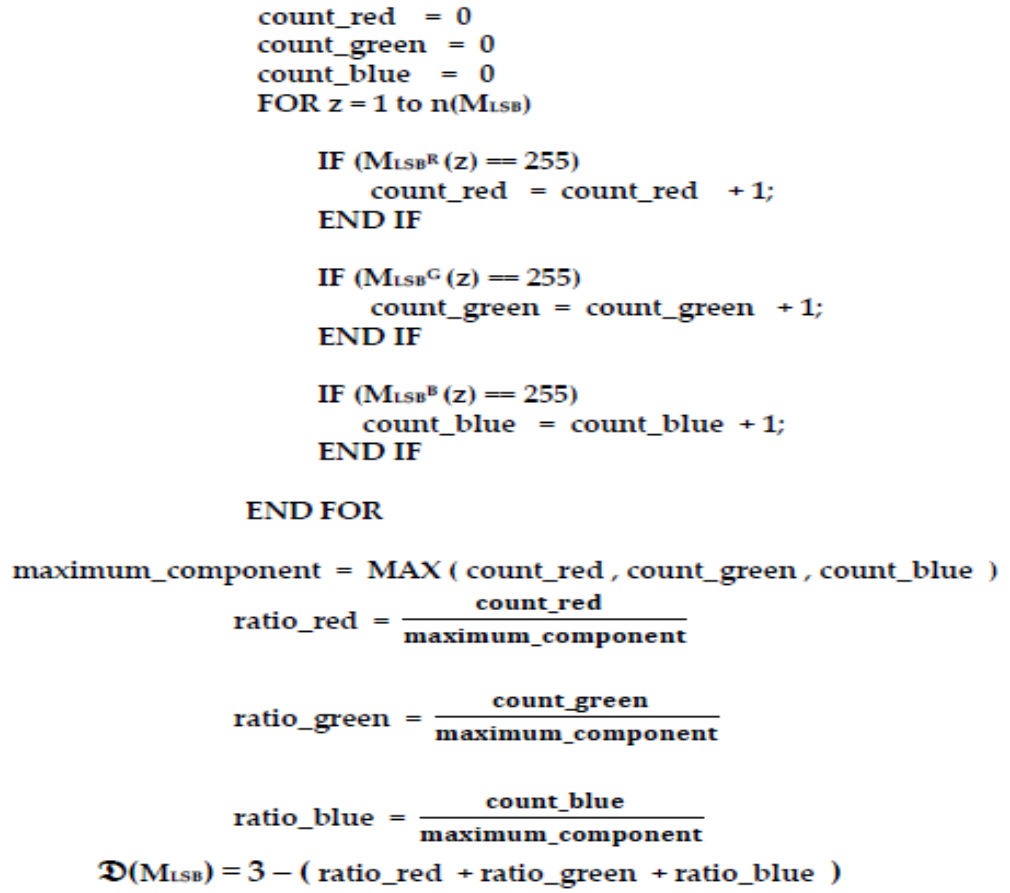

\section{Definition 7 (Gödel Color Code of a Pixel Color in Multicolored LSB Transform)}

Gödel Code of any symbol is a unique number corresponding to the symbol. Since any pixel in the Multicolored LSB Transform of any image can have only of the seven possible colors (R, G, B, R+G, R+B, $\mathrm{B}+\mathrm{G}, \mathrm{R}+\mathrm{G}+\mathrm{B}$ and Black (absence of all the three $\mathrm{R}, \mathrm{G}$ and $\mathrm{B}$ ) as mentioned in Section 2.2.2 of [7]. Thus we can uniquely identify any pixel color in Multicolored LSB Transform by a unique numeric code called in this text as Gödel Color Code. We represent this Gödel Color Code for the color of the pixel $\mathrm{M}_{\mathrm{LSB}}(\mathrm{z})$ by $\varphi\left(\mathrm{M}_{\mathrm{LSB}}(\mathrm{z})\right)$.

$\varphi\left(\mathrm{M}_{\mathrm{LSB}}(\mathrm{z})\right)$ is determined by associating every pixel's $\mathrm{R}, \mathrm{G}$ and $\mathrm{B}$ value by a unique set of three different numbers i.e a unique triplet of numbers $\left\langle\mathrm{W}_{\mathrm{R}}, \mathrm{W}_{\mathrm{G}}, \mathrm{W}_{\mathrm{B}}>\right.$ acting as $\mathrm{RGB}$ _Identifiers. These numbers are such that the sum of any two numbers is different from any of the three numbers or the sum of any other two numbers or the sum of all the three numbers (eg: $<1,2,5>$ or $<1,2,4>$ (this triplet is used in chmod command of Unix based systems for directory $\mathrm{r}$ - $\mathrm{w}$-x permissions and has similar purpose) but not $\langle 1,2,3\rangle$ because $\quad 1+2=$ 3). Since every pixel is made up of the combination of the R, G and B values so by RGB _Identifiers each pixel color can be uniquely identified by a unique number. In other words the Gödel Color Code for any pixel in Multi Colored LSB Transform of an image can be obtained using RGB _Identifiers by adding only those values of $\mathrm{W}_{\mathrm{R}}, \mathrm{W}_{\mathrm{G}}, \mathrm{W}_{\mathrm{B}}$ in the RGB _Identifier corresponding to which the RGB colors are present in the pixel. Mathematically this can be explained as:

$$
\begin{aligned}
& \text { let Multicolored LSB Transform of image } M \text { is } M_{L S B} \\
& \text { RGB_Identifiers }=<W_{R}, W_{C}, W_{B}> \\
& \text { where } W_{R}, W_{G}, W_{B} \text { are such that: } \\
& W_{R} \neq W_{G} \neq W_{B} \neq W_{R}+W_{C} \neq W_{B}+W_{R} \neq W_{G}+W_{B} \neq W_{R}+W_{G}+W_{B} \\
& \varphi\left(M_{L S B}(z)\right)=\frac{M_{L S B}^{R}(Z)}{255} W_{R}+\frac{M_{L S B}^{G}(Z)}{255} W_{G}+\frac{M_{L S B}^{B}(Z)}{255} W_{B}
\end{aligned}
$$

\section{Definition 8 (Pixel Difference in Multicolored LSB Transform)}

Two pixels in the Multicolored LSB Transform of the image M are said to be different if they are differently colored. The pixel difference is a function which determines whether two pixels are having same colors or are differently colored. If the two pixels are differently colored then it produces value 1 and if they are same it produces a 0 value. The Pixel Difference of two pixels $\mathrm{M}_{\mathrm{LSB}}(\mathrm{y})$ and $\mathrm{M}_{\mathrm{LSB}}(\mathrm{z})$ is represented as $\vartheta\left(\mathrm{M}_{\mathrm{LSB}}(\mathrm{y})\right.$, $\left.\mathrm{M}_{\mathrm{LSB}}(\mathrm{z})\right)$ and its value is one if the difference of $\varphi\left(\mathrm{M}_{\mathrm{LSB}}(\mathrm{y})\right)$ and $\varphi\left(\mathrm{M}_{\mathrm{LSB}}(\mathrm{z})\right)$ is non-zero otherwise the Pixel Difference $\vartheta\left(\mathrm{M}_{\mathrm{LSB}}(\mathrm{y}), \mathrm{M}_{\mathrm{LSB}}(\mathrm{z})\right)$ is zero. Mathematically the Pixel Difference function $\vartheta$ in Multicolored LSB Transform is explained in (8) as:

$$
\vartheta\left(\mathrm{M}_{\mathrm{LSB}}(\mathrm{y}), \mathrm{M}_{\mathrm{LSB}}(\mathbf{z})\right)=\vartheta\left(\mathrm{M}_{\mathrm{LSB}}(\mathbf{z}), \mathrm{M}_{\mathrm{LSB}}(\mathbf{y})\right)=\left\{\begin{array}{c}
1 \text { if } \varphi\left(\mathrm{M}_{\mathrm{LSB}}(\mathbf{y})\right)-\varphi\left(\mathrm{M}_{\mathrm{LSB}}(\mathbf{z})\right) \neq 0 \\
\text { else } \\
\left.0 \text { if } \varphi\left(\mathrm{M}_{\mathrm{LSB}}(\mathbf{y})\right)-\varphi\left(\mathrm{M}_{\mathrm{LSB}}(\mathbf{z})\right)=\mathbf{0}_{\mathbf{8}}\right)
\end{array}\right.
$$




\section{Definition 9 (Pixel Anomaly in the Multicolored LSB Transform)}

In [6] it was shown that any image's Multicolored LSB Transform can be broadly classified as Fine Grained, Coarse Grained, Boulder Grained and Continuous Grained. This classification was based on the degree of difference of any particular pixel in the Multicolored LSB Transform of the image from the immediately neighboring pixels. The immediate neighbors of any pixel $\mathrm{M}_{\mathrm{LSB}}(\mathrm{z})$ is the set of neighboring pixels of $\mathrm{M}_{\mathrm{LSB}}(\mathrm{z})$ obtained using locality function $\ell\left(\mathrm{M}_{\mathrm{LSB}}(\mathrm{z})\right)$ with $\lambda=1$ and distance function $d$ calculating the Chess Board Distance (Definition 4, (4)). This degree of difference of any particular pixel in the Multicolored LSB Transform of the image from the immediately neighboring pixels is called Pixel Anomaly of the given pixel $\mathrm{M}_{\mathrm{LSB}}(\mathrm{z})$. Though logically the concept of Pixel Anomaly is similar to the concept of Pixel Aberration (Section 2.1 of [7] and Definition 7 in Section 2.2 of [6]) but since the Multicolored LSB Transform of any image has only 7 possible colors where as any normal image can have $256 \times 256 \times 256$ colors so the concept of Pixel Aberration (which is based on standard deviation) does not apply satisfactorily in the given case. A more specific method for determining the Pixel Anomaly in any Multicolored LSB Transform of the image is obtained using Pixel Difference function $\vartheta$ (Definition 8). The value of Pixel Anomaly for any particular pixel is said to be 1 if it is different from every other pixel in its immediate neighborhood where as its value is 0 when the given pixel is same (in color) as all its eight immediate neighboring pixels. Any value in between 1 and 0 indicates some of the pixels (the number of pixels depends on the magnitude of the value) in the neighborhood are different from the pixel concerned.

Thus Pixel Anomaly of any pixel in the multicolored LSB Transform of the Image is obtained by determining the mean of the modulus of the Pixel Differences of the concerned pixel from its immediate neighbors (locality function $\ell\left(\mathrm{M}_{\mathrm{LSB}}(\mathrm{z})\right)$ with $\lambda=1$ and distance function $d$ calculating the Chess Board Distance). Mathematically the Pixel Anomaly of any pixel $\mathrm{M}_{\mathrm{LSB}}(\mathrm{z})$ in multicolored LSB Transform of the Image $M$ is represented as $\boldsymbol{\Omega}\left(\mathrm{M}_{\mathrm{LSB}}(\mathrm{z}), \ell\left(\mathrm{M}_{\mathrm{LSB}}(\mathrm{z})\right)\right)$ and given in (9) as :

$$
\begin{aligned}
& \{(\mathrm{M}(\mathrm{z}))=\{\mathrm{M}(\mathrm{x}): \mathrm{M}(\mathrm{x}) \in \mathrm{M} \text { and } d(\mathrm{M}(\mathrm{x}), \mathrm{M}(\mathrm{z})) \leq \boldsymbol{\lambda}\} \\
& \text { Where distance function d calculates } \\
& \text { the Chess Board Distance and } \lambda=1 \text {. } \\
& \boldsymbol{\Omega}\left(\mathbf{M}_{\mathrm{LSB}}(\mathrm{z}), \ell\left(\mathrm{M}_{\mathrm{LSB}}(\mathrm{z})\right)\right)=\frac{1}{\mathbf{8}} \sum_{\substack{\mathrm{p} \in \ell\left(\mathrm{M}_{\mathrm{LSB}}(\mathrm{z})\right) \\
\mathrm{M}_{\mathrm{LSB}}(\mathrm{z}) \in \mathrm{M}_{\mathrm{LSB}}}} \vartheta\left(\mathrm{M}_{\mathrm{LSB}}(\mathrm{z}), \mathrm{p}\right)
\end{aligned}
$$

\section{Definition 10 (Mean Pixel Anomaly of the Multicolored LSB Transform)}

The Mean Pixel Anomaly $\boldsymbol{\Omega}\left(\mathrm{M}_{\mathrm{LSB}}\right)$ of a Multicolored LSB Transform $\mathrm{M}_{\mathrm{LSB}}$ of an Image $\mathrm{M}$ is the mean of the pixel anomalies of all the pixels in the given Multi Color LSB Transform of the image. Its value is said to be 1 when every pixel in the image is different from its immediate neighborhood. The value of $\boldsymbol{\Omega}\left(\mathrm{M}_{\mathrm{LSB}}\right)$ for the Image $\mathrm{M}_{\mathrm{LSB}}$ is given in (10) as:

$$
\Omega\left(M_{L S B}\right)=\frac{1}{n\left(M_{L S B}\right)} \sum_{\substack{z=1 \\ M_{L S B}(z) \in M_{L S B}}}^{z=n\left(M_{L S B}\right)} \Omega\left(M_{L S B}(z), \ell\left(M_{L S B}(z)\right)\right)
$$

\section{Definition 11 (Zero Count of Pixel Anomaly of the Multicolored LSB Transform)}

The Zero Count of Pixel Anomaly $\boldsymbol{z}\left(\mathrm{M}_{\mathrm{LSB}}\right)$ of a Multicolored LSB Transform $\mathrm{M}_{\mathrm{LSB}}$ of an Image $\mathrm{M}$ is the measure of the number of pixels in $\mathrm{M}_{\mathrm{LSB}}$ having Pixel Anomaly value as zero per unit pixel of $\mathrm{M}_{\mathrm{LSB}}$. I.e. it is the measure of the percentage of pixels with absolutely no difference in color with all the eight immediately neighboring pixels in $\mathrm{M}_{\mathrm{LSB}} . \boldsymbol{z}\left(\mathrm{M}_{\mathrm{LSB}}\right)$ is explained mathematically as:

$$
\text { zero }=0
$$

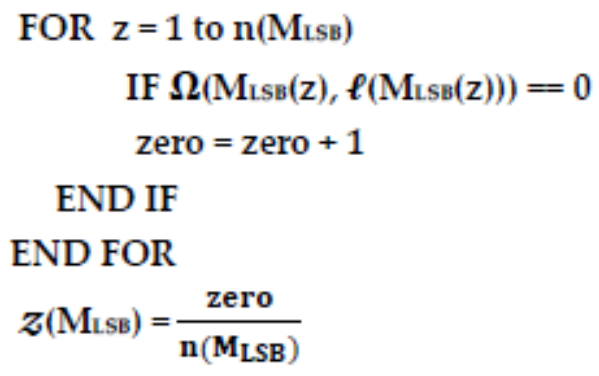




\section{Definition 12 (One Count of Pixel Anomaly of the Multicolored LSB Transform)}

The One Count of Pixel Anomaly $\boldsymbol{O}\left(\mathrm{M}_{\mathrm{LSB}}\right)$ of a Multicolored LSB Transform $\mathrm{M}_{\mathrm{LSB}}$ of an Image $\mathrm{M}$ is the measure of the number of pixels in $\mathrm{M}_{\mathrm{LSB}}$ having Pixel Anomaly value as absolutely one per unit pixel of $\mathrm{M}_{\mathrm{LSB}}$. I.e. it is the measure of the percentage of pixels which are having all its eight immediate neighbors absolutely different (in color) from the given pixel in $\mathrm{M}_{\mathrm{LSB}} \cdot \boldsymbol{\mathcal { O }}\left(\mathrm{M}_{\mathrm{LSB}}\right)$ is explained mathematically as:

$$
\text { one }=0
$$

FOR $\mathrm{z}=1$ to $\mathrm{n}$ (MLSB)

$$
\begin{aligned}
& \text { IF } \boldsymbol{\Omega}\left(\mathrm{MLSB}_{\mathrm{LB}}(\mathrm{z}), \boldsymbol{\ell}\left(\mathrm{M}_{\mathrm{LSB}}(\mathrm{z})\right)\right)=1 \\
& \text { one }=\text { one }+1 \\
& \text { END IF } \\
& \text { END FOR } \\
& \boldsymbol{O}\left(\mathrm{M}_{L S B}\right)=\frac{\text { one }}{\mathrm{n}\left(\mathrm{M}_{\mathrm{LSB}}\right)}
\end{aligned}
$$

\section{ANAlysis OF PiXels In LSB Planes AND Their Properties}

Multi-color LSB Planes of images can be classified as Fine Grained, Coarse Grained, Boulder Grained and Continuous Grained[6]. The same is shown in Figure 3. In certain cases the fine grained pixels may get combined with Continuous or Boulder grained pixels in the same sample and such clusters are known as Complex Grained Clusters. The image suffers degradation in it because of embedding of information in them and thus certain pixels gets modified corresponding to information are known as information pixels. Such modified pixels have maximum concentration in the multicolor LSB Transforms of the Stego Image. Therefore in addition the information pixels although are fine grained but are separately considered as a separate category of Information Pixels.
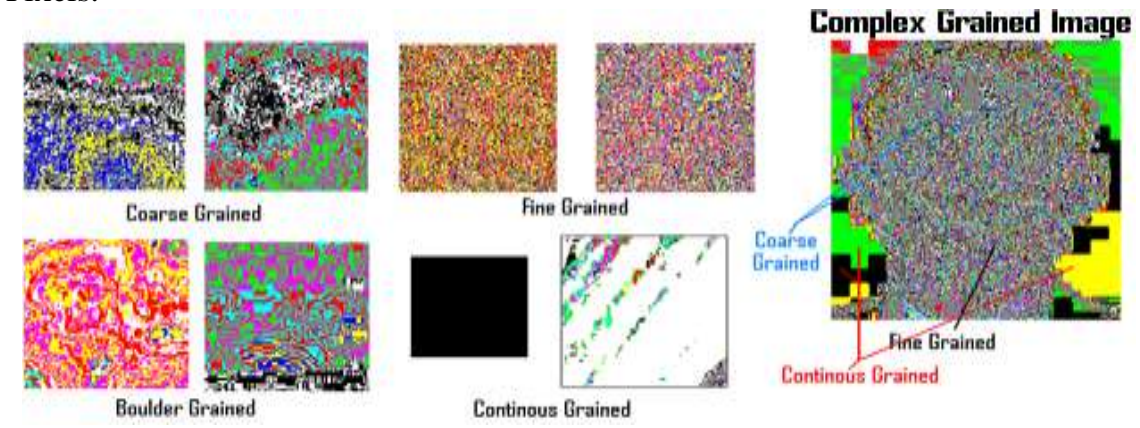

Fig 3 Types of Pixel Clusters in the Multicolored LSB Transform of any Image

The entire aim of this paper is to determine the properties of such information pixels so that they can be easily separated from the regular image components. Thus the Deviation from equal distribution of R:G:B given as $\mathfrak{D}\left(\mathrm{M}_{\mathrm{LSB}}\right)$ in definition 6, Mean Pixel Anomaly of the Multicolored LSB Transform (Definition 10) represented as $\Omega\left(\mathrm{M}_{\mathrm{LSB}}\right)$ in (10), Zero Count of Pixel Anomaly of the Multicolored LSB Transform) (Definition 11) represented as $z\left(\mathrm{M}_{\mathrm{LSB}}\right)$ in (11), One Count of Pixel Anomaly of the Multicolored LSB Transform $\mathcal{O}\left(\mathrm{M}_{\mathrm{LSB}}\right)$ in Definition 12 and ratio of $z\left(\mathrm{M}_{\mathrm{LSB}}\right)$ to $\mathcal{O}\left(\mathrm{M}_{\mathrm{LSB}}\right)$ is determined for Information Pixels and Innocent Pixels. In

\begin{tabular}{|c|c|c|c|c|c|}
\hline $\mathbf{M}_{\text {LSB }}$ & $D\left(M_{2 \leq n}\right)$ & $\Omega\left(\mathbf{I}_{2 \leq n}\right)$ & $=\left(\mathbf{M}_{\mathbf{L}}\right)$ & $o\left(M_{1, s n}\right)$ & $\frac{\mathrm{z}}{\partial}\left(\mathbf{M}_{\mathrm{L}, \mathrm{B}}\right)$ \\
\hline & 0.0337 & 0.7681 & 0 & 0.1488 & 0 \\
\hline & 0.0417 & 0.5978 & 0.0096 & 0.0360 & 0.2667 \\
\hline & 0.1174 & 0.6624 & 0 & 0.0667 & 0 \\
\hline & 0.0604 & 0.616 .4 & 0.0024 & 0.0300 & 0.0800 \\
\hline & 0.0819 & 0.8236 & 0 & 0.2144 & 0 \\
\hline Me & 0.06702 & 0.69366 & 0.0024 & 0.09918 & 0.06934 \\
\hline
\end{tabular}
Table 1 these five parameters are calculated for multicolor LSB Transforms of Fine Grained Innocent Pixel Clusters. Whereas in Table 2 the same parameters are calculated for multicolor LSB Transforms of Information Pixels which are even though fine grained but still are different from Innocent Pixels. In Table 3 and Table 4 the same values are calculated for Continuous and Boulder Grained pixels respectively. In Table 5 the Complex grained Pixels are analyzed. 


\begin{tabular}{|c|c|c|c|c|c|c|}
\hline $\mathbf{M}_{\mathrm{LSB}}$ & $\begin{array}{l}\text { DESCRIPTIOO } \\
N\end{array}$ & $D\left(\mathbf{M}_{\mathbf{L} S \mathrm{~B}}\right)$ & $\rho\left(\mathbf{M}_{\mathbf{L} \backslash \mathrm{B}}\right)$ & $z\left(\mathbf{M}_{\text {LSB }}\right)$ & $o\left(\mathbf{M}_{\mathrm{LSB}}\right)$ & $\frac{3}{\sigma}\left(\mathbf{M}_{\mathrm{LSB}}\right)$ \\
\hline & $\begin{array}{l}\text { If } \\
\text { Paragraph } \\
\text { of this } \\
\text { paper }\end{array}$ & 0.7740 & 0.7935 & o & 0.2460 & 0 \\
\hline a & $\begin{array}{l}2^{\text {and }} 3^{\text {ra }} \\
\text { Paragraph } \\
\text { of this } \\
\text { paper }\end{array}$ & 0.8467 & 0.8041 & 0 & 0.2308 & 0 \\
\hline & $\begin{array}{l}\text { Repeated } \\
\text { sequence or } \\
\text { a..... }\end{array}$ & 1.1878 & 0.6760 & 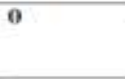 & 0.0452 & 0 \\
\hline & $\begin{array}{l}\text { Repeated } \\
\text { sequence of } \\
\text { abc....z1234 }\end{array}$ & 0.5771 & 0.8376 & o & 0.3450 & 0 \\
\hline & $\begin{array}{l}\text { Repeated } \\
\text { sequence of } \\
\text { abcd....abcd }\end{array}$ & 0.9518 & 0.7956 & o & 0.2052 & 0 \\
\hline Mean & & 0.86748 & 0.78136 & $\theta$ & 0.2144 & 0 \\
\hline
\end{tabular}

Table 2 Properties of Information Pixels

\begin{tabular}{|c|c|c|c|c|c|}
\hline $\mathbf{M}_{\mathrm{L} \backslash \mathrm{SH}}$ & D $\left(\mathrm{M}_{\mathbf{L} \times w}\right)$ & $\Omega\left(M_{L S B}\right)$ & $z\left(\mathbf{M}_{\mathrm{LSB}}\right)$ & $o\left(\mathbf{M}_{L<8}\right)$ & $\frac{z}{o}\left(\mathbf{M}_{\mathbf{L} \leq \mathrm{x}}\right)$ \\
\hline & 0.3128 & 0.1841 & 0.5520 & 0.0052 & 106.1538 \\
\hline & 0.4944 & 0.0770 & 0.7508 & 0.0016 & 469.2500 \\
\hline & 0.1507 & 0.2223 & 0.4832 & 0.0048 & 100.6667 \\
\hline & 1.7991 & 0.0831 & 0.7524 & 0.0044 & 171.0000 \\
\hline & 0.0027 & 0.1344 & 0.6288 & 0.0020 & 314.4000 \\
\hline Mean & 0.55194 & 0.14018 & 0.6538 & 0.0036 & 232.2941 \\
\hline
\end{tabular}

Table 3 Properties of Continuous Grained Pixels

\begin{tabular}{|c|c|c|c|c|c|}
\hline $\mathrm{M}_{\mathrm{LSn}}$ & $D\left(\mathbf{M}_{L S B}\right)$ & $\Omega\left(\mathbf{M}_{L S B}\right)$ & $\approx\left(M_{L S B}\right)$ & $o\left(\mathrm{M}_{\mathrm{LSB}}\right)$ & $\frac{5}{\partial}\left(\mathbf{M}_{\mathbf{L S B}}\right)$ \\
\hline & 0.1474 & 0.3494 & 0.1291 & 0.0089 & 14.4615 \\
\hline & 1.5642 & 0.3227 & 0.1335 & $4.3478 \mathrm{e}-004$ & 307 \\
\hline & 0.0706 & 0.4574 & 0.1480 & 0.0220 & 6.7273 \\
\hline & 0.0311 & 0.3227 & 0.1335 & $8.6957 e-004$ & 153.5000 \\
\hline & 0.1567 & 0.3229 & 0.2238 & 0.0035 & 64.6667 \\
\hline Mean & 0.394 & 0.35502 & 0.15358 & 0.007141 & 109.2711 \\
\hline
\end{tabular}

\begin{tabular}{|c|c|c|c|c|c|}
\hline $\mathbf{I}_{\text {LSB }}$ & $D\left(\mathbf{M}_{1,58}\right)$ & $\Omega\left(M_{L} \leq B\right)$ & $=\left(\mathbf{I}_{\mathrm{L} S \mathrm{~B}}\right)$ & $o\left(\mathbf{M}_{\mathrm{LSB}}\right)$ & $\frac{z}{\partial}\left(\mathbf{M}_{\text {LSB }}\right)$ \\
\hline & 0.4618 & 0.5583 & 0.1244 & 0.0892 & 1.3946 \\
\hline & 0.2410 & 0.5060 & 0.2352 & 0.0580 & 4.0552 \\
\hline & 0.9695 & 0.5585 & 0.1836 & 0.1124 & 1.6335 \\
\hline & 0.0271 & 0.4329 & 0.3624 & 0.1016 & 3.5669 \\
\hline & 0.1005 & 0.5100 & 0.1843 & 0.0820 & 2.2488 \\
\hline Mean & 0.35998 & 0.51314 & 0.21798 & 0.08864 & 2.5798 \\
\hline
\end{tabular}

\section{Conclusion}

From Table 1, Table 2, Table 3, Table 4 and Table 5 it can be concluded that Information Pixels as well as the Continuous grained pixels have large value of deviation from equal distribution of Red, Green and Blue Components (R:G:B = 1:1:1). In other words the value of $\mathfrak{D}\left(\mathrm{M}_{\mathrm{LSB}}\right)$ for Information Pixels as well as the Continuous Grained Pixels is very high (Average is 0.86748 and 0.55194 respectively). The $\mathfrak{D}\left(\mathrm{M}_{\mathrm{LSB}}\right)$ of Innocent Fine Grained Pixel Clusters is very low (Average is 0.06702). Interestingly the $\mathfrak{D}\left(\mathrm{M}_{\mathrm{LSB}}\right)$ of Complex and Boulder Grained pixels may vary from very low to very high values. This is because the degree of deviation $\mathfrak{D}$ in boulder grained and complex components depends upon the constituent colors forming the boulder grained or Complex Grained Pixel cluster. For example the boulder grained image component in $4^{\text {th }}$ row of Table 4 is composed of colors Green and Magenta (which in turn is Red + Blue) causing almost equal distribution of Red, 
Green and Blue components in the image causing degree of deviation to be close to zero (0.0311) but it is not necessary that $\mathfrak{D}$ value will be close to zero for all boulder grained or Complex Grained pixel clusters.

In addition to high value of degree of deviation the continuous grained pixels always have high value of Zero Count (0.6538) but lower values of pixel anomaly (0.14018) and One Count $(0.0036)$. Thus the value of the ratio of Zero Count to One Count $z / \mathcal{O}$ is higher (232.2941) in continuous grained components. The reason for this is that all the pixels in Continuous grained clusters are primarily composed of same colored pixels causing high values of zero count $z$ and lower values of One Count $\mathcal{O}$ leading to reduction in pixel aberration $\Omega$ and hence are also biased towards any particular color causing higher deviation $\mathfrak{D}$. This applies not just on Continuous grained pixels but also on Boulder grained pixels (on an average the ratio of Zero Count to One Count $z / \mathcal{O}$ is higher 109.2711 and Pixel Anomaly $\Omega$ is in between high and low around 0.35502). But unlike continuous grained or Boulder Grained pixels the information pixels have higher values of Pixel Anomaly $\Omega$ (average is around 0.78136 ) and also the value of zero count $z$ is always zero (it was found to be absolutely zero for every other information pixel samples obtained from various stego algorithms including the information pixel samples of size $600 \times 600$ Pixels containing information pertaining to single characters like series of a...a, $\mathrm{b} \ldots \mathrm{b}$, and even $\mathrm{z} \ldots \mathrm{z}$ ) and has high values of one count $\mathcal{O}$ (average is 0.2144 ) thus causing the value of $z / \mathcal{O}$ to be exactly 0 . Thus from these 5 parameters the information pixels can be easily differentiated from the Continuous grained Pixels.

But even the innocent fine grained pixels may sometimes have zero value of zero count $z\left(1^{\text {st }}, 3^{\text {rd }}\right.$ and $5^{\text {th }}$ entry of Table 1) and always has high values of Pixel Anomaly $\Omega$ (average is around 0.69366) but still they can be easily isolated from the information pixels by the significantly high value of the degree of deviation from $\mathrm{R}: \mathrm{G}: \mathrm{B}=1: 1: 1 \mathfrak{D}$ (average is around 0.86748).

The complex pixel clusters may contain information in their fine grained sections. As a result they must be isolated from the rest of the image and must be analyzed in detail. The complex grained pixels can be identified by their average values of the ratio of Zero Count to One Count $z / \mathcal{O}$ (on an average 2.5798) but it may vary from 1 to 15 ( $1^{\text {st }}$ and $3^{\text {rd }}$ entry of table 4 (Boulder Grained Pixels) may also be classified as Complex Grained ). Mathematically this is summarized in (13).

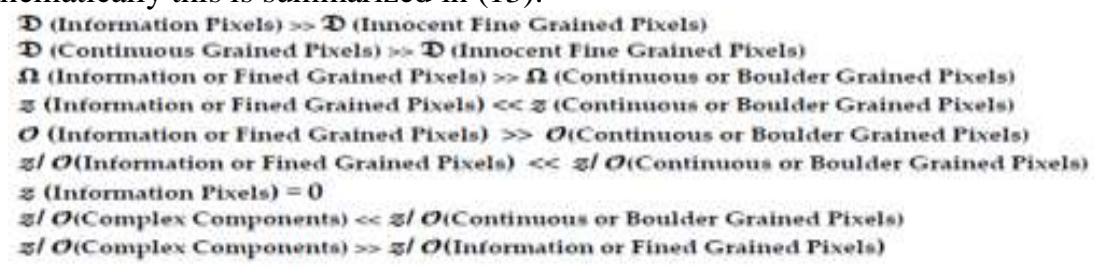

I wish to dedicate this work to my parents Mr Chandan Kumar Choudhary and Mrs Nilima Choudhary for providing the necessary support and encouragement in all walks of life including this work.

\section{REFERENCES}

[1] Kaustubh Choudhary, Image Steganography and Global Terrorism, IOSR Volume 1, Issue 2, July 2012.http://iosrjournals.org/journals/iosr-jce/papers/vol1-issue2/14/N0123448.pdf

[2] Infosecurity Magazine article dated 02 May 2012 reports that Al-Qaeda uses Steganography to hide documents. http://www.infosecurity-magazine.com/view/25524/alqaeda-uses-steganography-documents-hidden-in-porn-videos-found-onmemory-stick

[3] Daily Mail Online, UK article dated 01 May 2012 reported that a Treasure trove of Intelligence was embedded in porn video.http://www.dailymail.co.uk/news/article-2137848/Porn-video-reveals-Al-Qaeda-planns-hijack-cruise-ships-executepassengers.html\#ixzzluIgxpire

[4] The New York Times article dated Oct 30, 2001 with title "Veiled Messages of Terror May Lurk in Cyberspace" claims 9/11 attacks planned using Steganography.

[5] Wired article dated 02 $2^{\text {nd }}$ July, 2001 nicknamed Bin Laden as "the Steganography Master"

[6] Kaustubh Choudhary, Novel Approach to Image Steganalysis (A Step against Cyber Terrorism) IOSR Volume 2, Issue 5, August 2012http://iosrjournals.org/journals/iosr-jce/papers/vol2-issue5/B0251628.pdf

[7] Kaustubh Choudhary, Mathematical Modeling of Image Steganographic System IOSR Volume 2, Issue 5, August 2012http://iosrjournals.org/journals/iosr-jce/papers/vol2-issue5/A0250115.pdf

[8] Kaustubh Choudhary, Identification of Stego Signatures in Images using Suspicion Value (special reference to Concentrating Stego Algorithms), IOSR Volume 3, Issue 4, August 2012

[9] Kaustubh Choudhary, Quick Identification of Steganographic Signatures of Distributing Stego Algorithms using Suspicion Value, IOSR Volume 3, Issue 4, August 2012.

\section{ABOUT THE AUTHOR}

\begin{tabular}{|l|l|} 
& $\begin{array}{l}\text { Kaustubh Choudhary, Scientist, Defence Research and Development Organisation (DRDO)Ministry of Defence, } \\
\text { Government of India } \\
\text { Current Attachement: } \\
\text { Attached with Indian Navy at Naval College of Engineering, Indian Naval Ship Shivaji, Lonavla-410402, } \\
\text { Maharashtra,India }\end{array}$ \\
\hline
\end{tabular}

